\title{
Reduced Histone H3 Lysine 9 Methylation Contributes to the Pathogenesis of Latent Autoimmune Diabetes in Adults via Regulation of SUV39H2 and KDM4C
}

\author{
$\mathrm{Xi}-\mathrm{yu} \mathrm{Liu}^{1}$ and Hong $\mathrm{Li}^{1,2}$ \\ ${ }^{1}$ Department of Endocrinology, Fourth Affiliated Hospital of Zhejiang University School of Medicine, Yiwu, Zhejiang, China \\ ${ }^{2}$ Department of Endocrinology, Sir Run Run Shaw Hospital, School of Medicine, Zhejiang University, Hangzhou, Zhejiang, China \\ Correspondence should be addressed to Hong Li; syflihong@163.com
}

Received 25 October 2016; Revised 1 January 2017; Accepted 2 March 2017; Published 15 March 2017

Academic Editor: Paolo Fiorina

Copyright (C) $2017 \mathrm{Xi}$-yu Liu and Hong Li. This is an open access article distributed under the Creative Commons Attribution License, which permits unrestricted use, distribution, and reproduction in any medium, provided the original work is properly cited.

\begin{abstract}
Aims. Latent autoimmune diabetes in adults (LADA) is an autoimmune disease of which the mechanism is not clear. Emerging evidence suggests that histone methylation contributes to autoimmunity. Methods. Blood CD4 ${ }^{+} \mathrm{T}$ lymphocytes from 26 LADA patients and 26 healthy controls were isolated to detect histone $\mathrm{H} 3$ lysine 4 and $\mathrm{H} 3$ lysine 9 methylation status. Results. Reduced global H3 lysine 9 methylation was observed in LADA patients' $\mathrm{CD}^{+} \mathrm{T}$ lymphocytes, compared to healthy controls $(P<0.05)$. H3 lysine 4 methylation was not statistically different. The reduced H3 lysine 9 methylation was associated with GADA titer but not correlated with glycosylated hemoglobin (HbAlc). When the LADA patient group was divided into those with complication and those without, relatively reduced global H3 lysine 9 methylation was observed in LADA patients with complication $(P<0.05)$. The expression of histone methyltransferase SUV39H2 for $\mathrm{H} 3$ lysine 9 methylation was downregulated in LADA patients, and the expression of histone demethylase KDM4C which made $\mathrm{H} 3$ lysine 9 demethylation was upregulated. Conclusion. The reduction of histone $\mathrm{H} 3$ lysine 9 methylation which may due to the downregulation of methyltransferase SUV39H2 and the upregulation of demethylase KDM4C was found in $\mathrm{CD} 4^{+} \mathrm{T}$ lymphocytes of LADA patients.
\end{abstract}

\section{Introduction}

The prevalence of diabetes in Chinese adults was estimated to be $11.6 \%$ according to a survey in 2013 [1]. Latent autoimmune diabetes in adults (LADA) is a subtype of type 1 diabetes. The total prevalence of LADA was as high as $8.63 \%$ and GADA positive LADA was $5.9 \%$ in clinical newly diagnosed type 2 diabetes in China [2], so the number of Chinese LADA patients was large. LADA is an autoimmune disease characterized by T-cell-mediated immune destruction of the islet $\beta$-cells, and $\mathrm{CD}_{4}^{+} \mathrm{T}$ lymphocytes play a central role in the pathogenesis of LADA [3-6]. Although extensive evidence showed that LADA was an autoimmune disease, the exact molecular mechanism remains to be explored [7].
In the recent decades, histone methylation, which helped the genome expression, has become a research hotspot. Evidence has demonstrated that histone methylation played an important role in autoimmune diseases $[8,9]$. Recent research also showed that histone methylation played an important role in the pathogenesis of diabetes [10,11]. LADA was an autoimmune disease, so we hypothesized that histone methylation may associate with the pathogenesis of LADA.

Miao et al. studied histone modification in classical type 1 diabetes patients. They found that lymphocytes from classical type 1 diabetes patients displayed distinct profile of chromatin histone 3 lysine 9 dimethylation [12], and $\mathrm{H} 3$ lysine 9 acetylation levels were markedly varied in classical type 1 diabetes monocytes relative to controls [13]. 


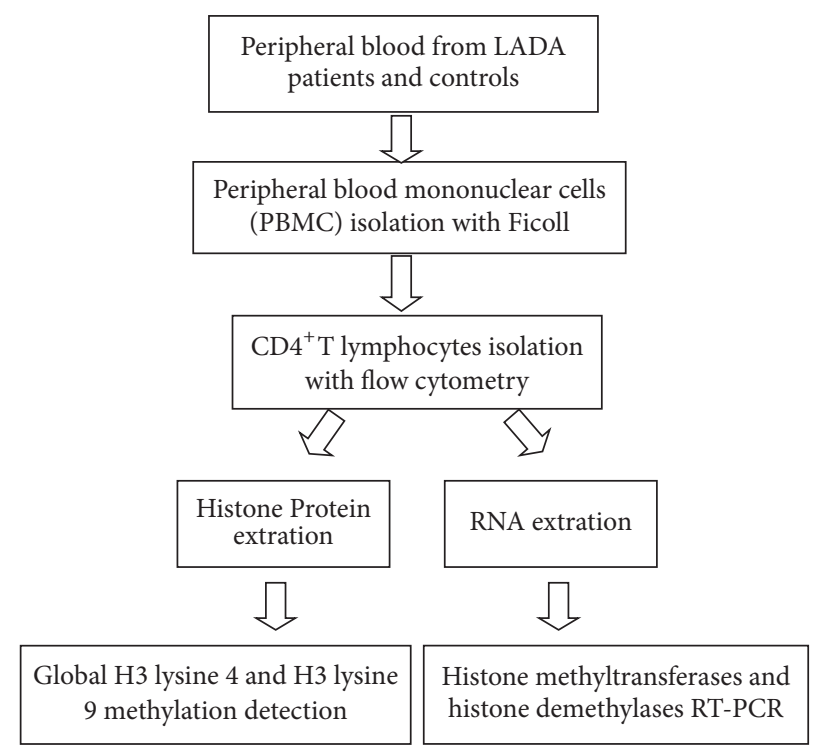

FIgure 1: The study scheme. Twenty-six LADA patients and 26 healthy control subjects were enrolled in this study. Peripheral blood mononuclear cells (PBMC) were isolated with Ficoll from peripheral venous blood which was obtained from each participant. CD4 ${ }^{+} \mathrm{T}$ lymphocytes were isolated by flow cytometry. Then, Global H3 lysine 4 and H3 lysine 9 methylation was detected in CD $4^{+}$T lymphocytes from 26 subjects. The mRNA level of histone methyltransferases gene (SET1, SUV39H1, SUV39H2, and G9a) and histone demethylases gene (LSD1, KDM3A, KDM4A, KDM4B, KDM4C, KDM5A, KDM5B, KDM5C, and KDM5D) was detected by real-time PCR.

Type 1 diabetes is divided into two subtypes (classical type 1 diabetes and LADA) [14, 15]. The present study of histone methylation in type 1 diabetes patients focused on classical type 1 diabetes, rarely in LADA. Therefore, it is important to perform research of histone methylation in LADA. The present study of histone methylation in type 1 diabetes chose lymphocytes or monocytes, but $\mathrm{CD} 4^{+} \mathrm{T}$ lymphocytes play a central role in the pathogenesis of LADA. Therefore, we investigated the global histone methylation pattern and the gene expression status of specific histone methyltransferases and demethylases in $\mathrm{CD} 4^{+} \mathrm{T}$ lymphocytes from LADA patients and healthy controls.

\section{Methods}

2.1. Patients and Controls. We enrolled 52 volunteers with 26 LADA patients and 26 healthy controls. Patients who were considered to have had LADA with age above 30 years old on the basis of glutamic acid decarboxylase autoantibody (GADA) positive were eligible to undergo this study if they had no history of tumor, other immune disease. The controls were considered to be healthy on the basis of normal oral glucose tolerance test (OGTT) and negative GADA if they had no history of immune disease, diabetes, or tumors. The LADA patients of this study included 12 patients absent of diabetic complication and 14 patients with at least one microvascular or macrovascular complication, specifically, 8 patients with retinopathy, 3 patients with neuropathy, 6 patients with nephropathy, and 4 patients with macrovascular complication. GADA positivity above or below 180 units $/ \mathrm{ml}$ was the accepted threshold to divide LADA into two groups, with high GADA titer ( $\geq 180$ units/ml) and low GADA titer ( $<180$ units $/ \mathrm{ml}$ and $\geq 18$ units/ml). Experimental scheme of this study was shown in Figure 1. Our research was in compliance with the Helsinki Declaration. The ethics approval and consent were approved by the Ethics Committee of Sir Run Run Shaw Hospital, School of Medicine, Zhejiang University, and the Fourth Affiliated Hospital of Zhejiang University School of Medicine.

2.2. Cells Preparation. Peripheral venous blood was obtained from each patient and healthy control. Then, peripheral blood mononuclear cells were isolated by density gradient centrifugation using Ficoll gradients. $\mathrm{CD} 4^{+} \mathrm{T}$ lymphocytes were separated by flow cytometry and stored at $-80^{\circ} \mathrm{C}$. The antibodies used for cell sorting in the flow cytometry were as follows: FITC mouse anti-human CD3 clone antibodies and FITC mouse IgG1, $\kappa$ isotype control, APC-H7 mouse antihuman CD4 clone antibodies and APC-H7 mouse IgG1, $\kappa$ isotype control (BD Company, America).

2.3. Detection of Global H3 Lysine 4 and H3 Lysine 9 Methylation. Global H3 lysine 4 and H3 lysine 9 methylation were detected according to the manufacturer's instructions of the EpiQuik ${ }^{\mathrm{TM}}$ global histone $\mathrm{H} 3$ lysine 4 and $\mathrm{H} 3$ lysine 9 methylation assay kits (Epigentek, America). Cells were collected into a tube and the lysis buffer was used to lyse cells. Histone was extracted by using extraction buffer and TCA solution. Histone proteins of samples and the methylated $\mathrm{H} 3$ lysine 4 or $\mathrm{H} 3$ lysine 9 control were spotted on the strip wells. Capture antibody was added after wash. The methylated histone $\mathrm{H} 3$ lysine 4 or $\mathrm{H} 3$ lysine 9 was recognized with 
TABle 1: Primer sequences used in real-time PCR.

\begin{tabular}{lcc}
\hline Gene & Forward primer $\left(5^{\prime}-3^{\prime}\right)$ & Reverse primer $\left(5^{\prime}-3^{\prime}\right)$ \\
\hline SET1 & GACACAAGCTTCTCCAGCA & TGAAGATGCAGAGAAGTGGC \\
SUV39H1 & AAGAAGATCCGCGAACAGGA & GGAAGTGCTTGAGGATACGGAC \\
SUV39H2 & ATCCCACCTGGTACTCCCATCT & GCAAAGCGAATACTGTGTGCC \\
G9a & ACAAAGAGGGGGACACAGCA & ATGGTGGACGTCTCGCAGTT \\
LSD1 & TTCTGGAGGGTATGGAGACG & ACCTTCTGGGTCTGTTGTGG \\
KDM3A & GTGCTCACGCTCGGAGAAA & AAACAGCTCGAATGGTCCCG \\
KDM4A & AGAGTTCCGCAAGATAGCCAA & AGTCCAGGATTGTTCTCAGCC \\
KDM4B & CTTTGGGGAGCCTAAGTCCTG & GGTAGCCGTAGGGAAATGTGA \\
KDM4C & GAGGAGCTAGAGGCCAAGC & CTTCCAGGTCGGACCCTTC \\
KDM5A & CCGTCTTTGAGCCGAGTTG & GGACTCTTGGAGTGAAACGAAA \\
KDM5B & AAGAGACGCCTGGAAAGAGAG & CCAAACCTCACTGACCTCATC \\
KDM5C & GGGTCCGACGATTTCCTACC & GCGATGGGCCTGATTTTCG \\
KDM5D & GGGTCCGACGATTTCCTACC & GCGATGGGCCTGATTTTCG \\
$\beta$-ACTIN & GCACCA CAC CTT CTA CAA TGA GC & GGA TAG CACAGC CTG GATAGCAAC \\
\hline
\end{tabular}

TABLE 2: Characteristics of the participates.

\begin{tabular}{lcccc}
\hline \multirow{2}{*}{ Parameters } & \multirow{2}{*}{ LADA patients $(n=26)$} & Control subjects $(n=26)$ & \multicolumn{2}{c}{ LADA patients } \\
& & & Complication-free $(n=12)$ & With complication $(n=14)$ \\
\hline Gender (male\%) & $46.2 \%$ & $46.2 \%$ & $50.0 \%$ & $42.9 \%$ \\
Age (years) & $43 \pm 9$ & $39 \pm 9$ & $37 \pm 7$ & $48 \pm 10$ \\
BMI (kg/m ${ }^{2}$ ) & $23.9 \pm 3.6$ & $23.6 \pm 3.9$ & $23.7 \pm 4.1$ & $24.0 \pm 4.2$ \\
Diabetes duration (years) & $9 \pm 7$ & $0^{* *}$ & $5 \pm 5$ & $12 \pm 8^{\#}$ \\
HbAlc (\%) & $9.6 \pm 2.6$ & $5.2 \pm 0.3^{*}$ & $8.0 \pm 2.3$ & $12.1 \pm 2.8^{\#}$ \\
FCP (nmol/l) & $0.49 \pm 0.27$ & - & $0.62 \pm 0.32$ & $0.37 \pm 0.28$ \\
Low FCP & $8 / 26(30.8 \%)$ & - & $2 / 12(16.7 \%)$ & $6 / 14(42.9 \%)$ \\
High titer GADA & $9 / 26(34.6 \%)$ & - & $4 / 12(33.3 \%)$ & $5 / 14(35.7 \%)$ \\
Low titer GADA & $17 / 26(65.4 \%)$ & - & $8 / 12(66.7 \%)$ & $9 / 14(64.3 \%)$ \\
Total cholesterol (mmol/l) & $4.2 \pm 1.0$ & $4.3 \pm 1.0$ & $4.1 \pm 0.9$ & $4.3 \pm 1.1$ \\
Triglyceride (mmol/l) & $1.8 \pm 1.1$ & $1.9 \pm 1.2$ & $1.7 \pm 0.9$ & $1.9 \pm 1.4$ \\
HDL cholesterol (mmol/l) & $1.3 \pm 0.3$ & $1.2 \pm 0.3$ & $1.3 \pm 0.3$ & $1.3 \pm 0.2$ \\
LDL cholesterol (mmol/l) & $2.5 \pm 0.7$ & $2.4 \pm 0.8$ & $2.4 \pm 0.7$ & $2.5 \pm 0.8$ \\
Serum creatinine (umol/l) & $102 \pm 18.9$ & $80 \pm 17.2$ & $96 \pm 12.8$ & $108 \pm 16.7$ \\
\hline
\end{tabular}

$n$, number of subjects; BMI, body mass index; HbAlc, glycated hemoglobin; FCP, fasting C-peptide; HDL, high-density lipoprotein; LDL, low-density lipoprotein; GADA, glutamic acid decarboxylase antibody.

${ }^{*} P<0.05,{ }^{* *} P<0.01$, LADA patients compared with controls.

${ }^{\#} P<0.05$, LADA patients with complications compared with LADA patients without complications.

high-affinity antibody. The histone $\mathrm{H} 3$ lysine 4 or $\mathrm{H} 3$ lysine 9 methylation was quantified by calculating OD (sampleblank)/OD (untreated control-blank).

2.4. Real-Time PCR. Real-time PCR was performed using the one-step SYBR PrimeScript ${ }^{\mathrm{TM}}$ RT-PCR kit according to the manufacturer's instructions (Takara, Japan). $\beta$-Actin was used as an internal control to normalize for differences in the amount of total RNA of each sample. The histone methyltransferases (SET1, G9a, SUV39H1, and SUV39H2) and the histone demethylases (LSD1, KDM3A, KDM4A, KDM4B, KDM4C, KDM5A, KDM5B, KDM5C, and KDM5D) gene expression was detected by real-time quantitative PCR. Primer sequences of the above histone methylation modifier genes were listed in Table 1.
2.5. Statistical Analysis. Results were expressed as the mean \pm SD and analyzed using Student's $t$-test for continuous variables. Correlation was determined by Pearson's rank order correlation or Spearman's rank order correlation, depending on the normality of the data. $P<0.05$ were considered as significant. Real-time PCR data was analyzed by the comparative CT method (also named the $2^{-\Delta \Delta \mathrm{CT}}$ method). The absolute of fold change $>2$ was considered as significant.

\section{Results}

3.1. Characteristics of the Patients and Controls. The characteristics of the patients and healthy controls were shown in Table 2. There were no statistical differences in age, sex proportion, BMI, total cholesterol, triglyceride, HDL 


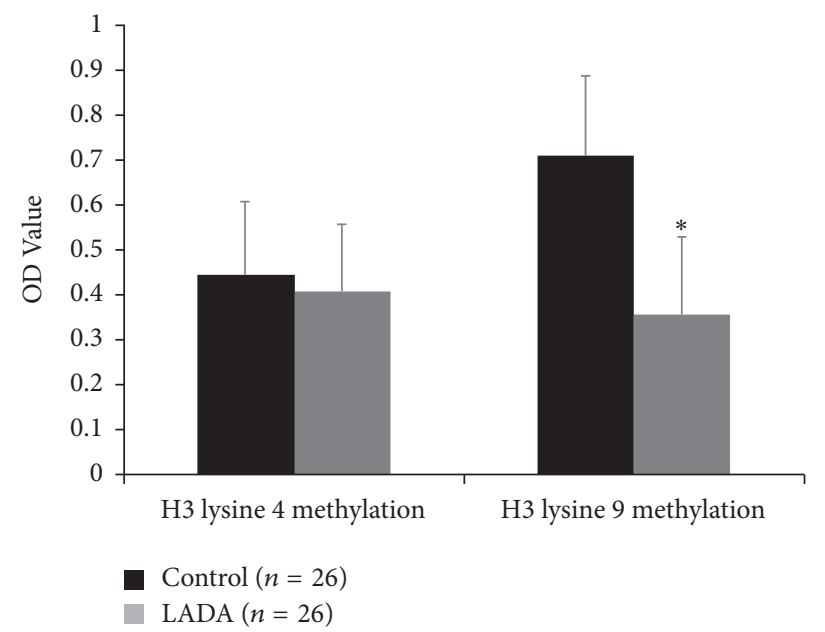

(a)

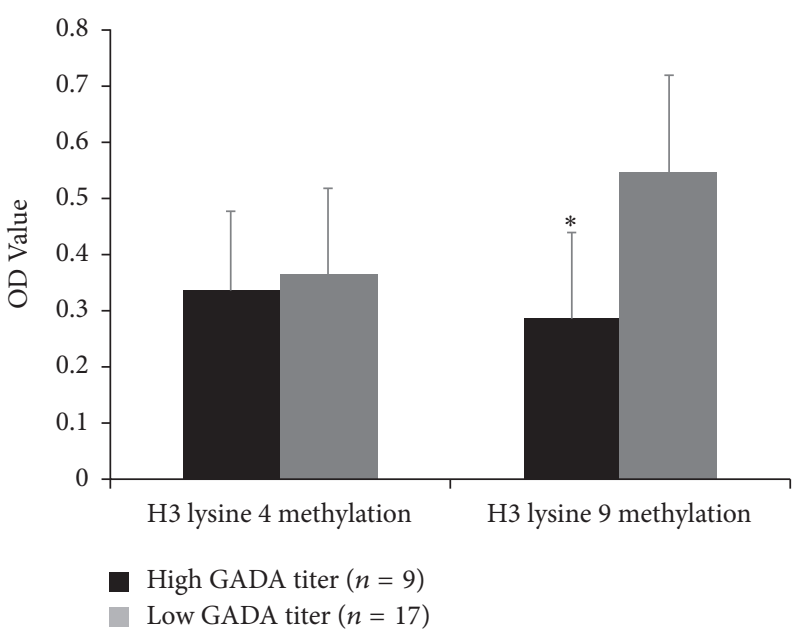

(b)

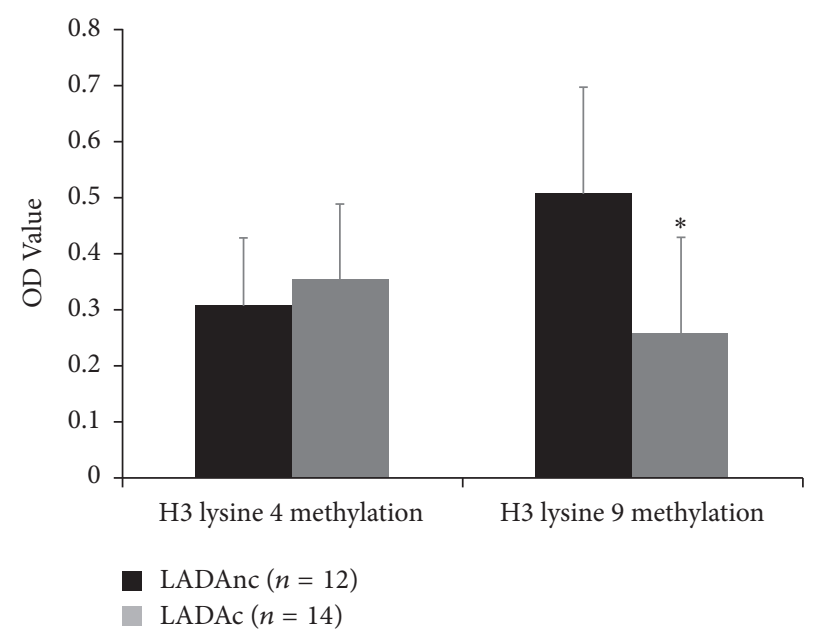

(c)

Figure 2: Reduced H3 lysine 9 methylation level in LADA patients. (a) Global H3 lysine 4 and H3 lysine 9 methylation level in CD4 ${ }^{+} \mathrm{T}$ lymphocytes from 26 LADA patients and 26 healthy controls, ${ }^{*} \mathrm{P}<0.05$, LADA patients versus healthy controls. (b) Global H3 lysine 4 and H3 lysine 9 methylation level in $\mathrm{CD} 4{ }^{+} \mathrm{T}$ lymphocytes from LADA patients, ${ }^{*} P<0.05$, LADA patients with low GADA titer ( $n=17$ ) versus LADA patients with high GADA titer $(n=9)$. (c) Global H3 lysine 4 and $\mathrm{H} 3$ lysine 9 methylation level in in CD $4^{+} \mathrm{T}$ lymphocytes from LADA patients, ${ }^{*} P<0.05$, LADA patients with complications (LADAc, $n=14$ ) versus LADA patients without complications (LADAnc, $n=12$ ).

cholesterol, LDL cholesterol, and serum creatinine between LADA patients group and healthy controls group $(P>0.05)$, but there were significant differences in HbAlc and diabetes duration between the two groups $(P<0.05)$. LADA group contained 12 patients without any diabetes complication and 14 patients with diabetes complications. When the LADA group was divided into those with complications and those without complication, there was statistical difference in HbAlc and diabetes duration between two subgroups $(P<$ $0.05)$.

3.2. Reduced H3 Lysine 9 Methylation in LADA Patients. We separated $\mathrm{CD}^{+} \mathrm{T}$ lymphocytes from 26 LADA patients and 26 healthy controls to investigate global histone $\mathrm{H} 3$ lysine 4 and H3 lysine 9 methylation levels. Reduced global H3 lysine
9 methylation was observed in $\mathrm{CD} 4^{+}$T lymphocytes of LADA patients compared to healthy controls $(P<0.05)$. However, $\mathrm{H} 3$ lysine 4 methylation level showed no statistical difference between the two groups (Figure 2(a)).

3.3. The Association between $\mathrm{H} 3$ Lysine 9 Methylation and GADA Titer. LADA was an autoimmune disease, and GADA positive was used to classify LADA. Every LADA patient in this study was GADA positive. We then compared the $\mathrm{H} 3$ lysine 9 methylation level between LADA patients with low GADA titer (18 units/ml $\leqslant$ GADA $<180$ units/ml) and LADA patients with high GADA titer (GADA $\geqslant 180$ units/ml). We found that reduced global $\mathrm{H} 3$ lysine 9 methylation level was observed in $\mathrm{CD}^{+}$T lymphocytes of LADA patients with high GADA titer $(P<0.05)$ (Figure $2(\mathrm{~b}))$. 
TABLE 3: Correlation analysis of global histone $\mathrm{H} 3$ lysine 4 and $\mathrm{H} 3$ lysine 9 methylation in $\mathrm{CD} 4^{+}$T lymphocytes from LADA patients.

\begin{tabular}{lccc}
\hline & \multicolumn{2}{c}{ H3 lysine 4 methylation $(n=26)$} & \multicolumn{2}{c}{ H3 lysine 9 methylation $(n=26)$} \\
& $r$ & $P$ & 0.376 \\
Age & 0.456 & 0.367 & 0.235 \\
BMI & 0.075 & 0.789 & 0.127 \\
HbAlc & -0.347 & 0.129 & -0.546 \\
FCP & 0.163 & 0.102 & 0.174 \\
Duration of Diabetes & 0.223 & 0.424 & 0.114 \\
Total cholesterol & 0.015 & 0.680 & -0.186 \\
Triglyceride & 0.074 & 0.823 & -0.235 \\
HDL cholesterol & 0.128 & 0.276 & -0.469 \\
LDL cholesterol & 0.217 & 0.289 & -0.159 \\
Serum creatinine & -0.136 & 0.212 & 0.1926 \\
GADA titer & -0.336 & 0.183 & 0.214 \\
\hline
\end{tabular}

H3 lysine 4 methylation and $\mathrm{H} 3$ lysine 9 methylation in LADA patients' $\mathrm{CD} 4^{+} \mathrm{T}$ lymphocytes were not correlated with age, BMI, HbAlc, FCP, duration of diabetes, total cholesterol, triglyceride, HDL cholesterol, LDL cholesterol, and serum creatinine. H3 lysine 9 methylation was associated with GADA titer. n, number of subjects; BMI, body mass index; HbAlc, glycated hemoglobin; FCP, fasting C-peptide; HDL, high-density lipoprotein; LDL, low-density lipoprotein; GADA, glutamic acid decarboxylase antibody.

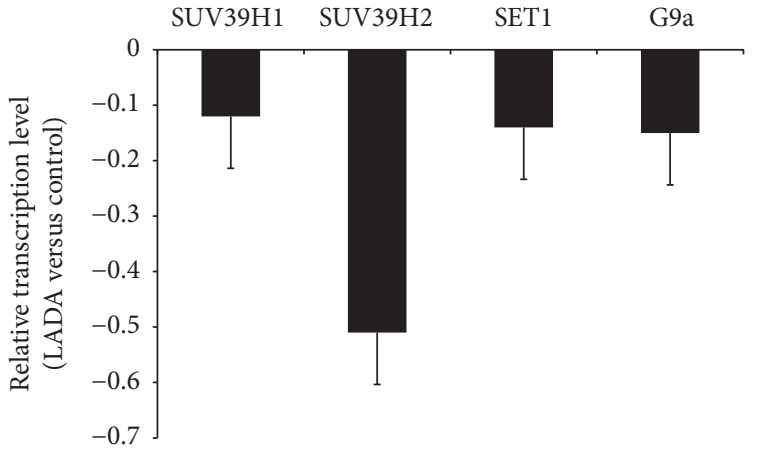

(a)

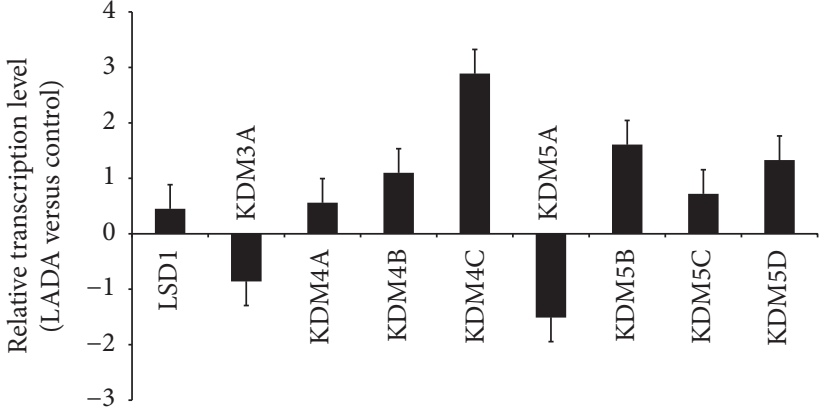

(b)

FIGURE 3: The mRNA level of histone methyltransferase and demethylase in $\mathrm{CD} 4^{+} \mathrm{T}$ lymphocytes from LADA patients. Data are normalized against beta-actin. The $\Delta$ Ct values were from 0.5 to 3.9. (a) Relative mRNA levels of histone methyltransferases (SET1, G9a, SUV39H1, and SUV39H2) in CD4 ${ }^{+}$T lymphocytes from LADA patients $(n=26)$ and healthy controls $(n=26)$, as measured by real-time PCR. The absolute of fold change $>2$ was considered significant, LADA patients versus healthy controls; data are normalized against beta-actin. (b) Relative mRNA levels of histone demethylases (LSD1, KDM3A, KDM4A, KDM4B, KDM4C, KDM5A, KDM5B, KDM5C, and KDM5D) in CD4 ${ }^{+} \mathrm{T}$ lymphocytes from LADA patients $(n=26)$ and healthy controls $(n=26)$, the absolute of fold change $>2$ was considered significant, LADA patients versus healthy controls; data are normalized against beta-actin.

3.4. H3 Lysine 9 Methylation Was Not Significantly Correlated with $\mathrm{HbAlc}$. We then analyzed the association between $\mathrm{H} 3$ lysine 9 methylation and clinical characteristics. We found that LADA CD4 ${ }^{+}$T lymphocytes $\mathrm{H} 3$ lysine 9 methylation was not related to $\mathrm{HbAlc}$, gender, age, $\mathrm{BMI}$, diabetes duration, fasting C-peptide, total cholesterol, triglyceride, HDL cholesterol, LDL-C cholesterol, and serum creatinine $(P>0.05)$, as seen in Table 3.

3.5. H3 Lysine 9 Methylation Associated with Complications of Diabetes. In order to assess whether $\mathrm{H} 3$ lysine 9 methylation was associated with complications of diabetes. We divided the LADA patients group into two subgroups, those with complications $(n=14)$ and those without complication $(n=12)$. We found that relatively reduced global H3 lysine 9 methylation $(P<0.05)$ was observed in $\mathrm{CD}^{+}$T lymphocytes of LADA patients with complications compared to those without complication (Figure 2(c)).

3.6. Gene Expression of Histone Methyltransferases and Demethylases. In order to investigate the cause of altered histone methylation patterns in LADA patients, we assessed mRNA levels of histone methyltransferases (SET1, G9a, SUV39H1, and SUV39H2) and the histone demethylases (LSD1, KDM3A, KDM4A, KDM4B, KDM4C, KDM5A, KDM5B, KDM5C, and KDM5D) in $\mathrm{CD}^{+}{ }^{+} \mathrm{T}$ lymphocytes by real-time quantitative $\mathrm{PCR}$.

As shown in Figure 3, we found that the expression of histone methyltransferase SUV39H2 in LADA patients was downregulated, respectively, compared to healthy controls (Figure 3(a)). The expression of histone demethylase KDM4C 


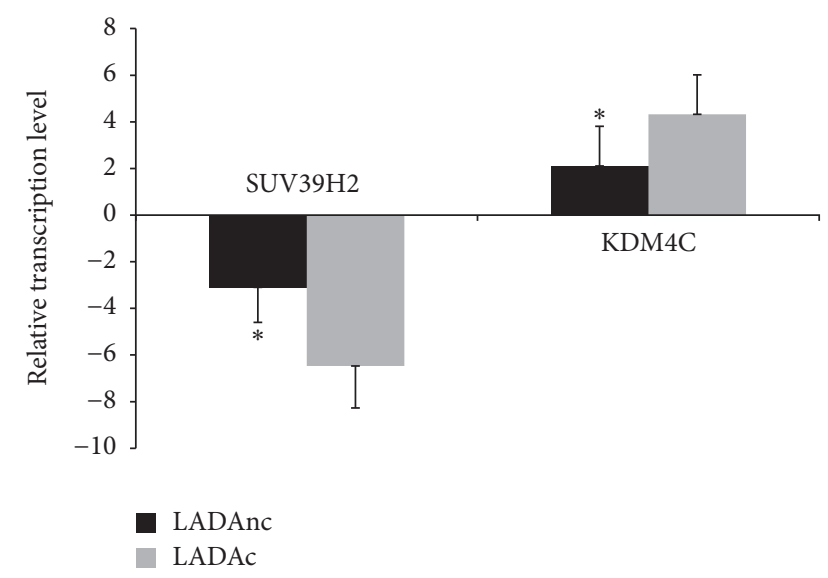

(a)

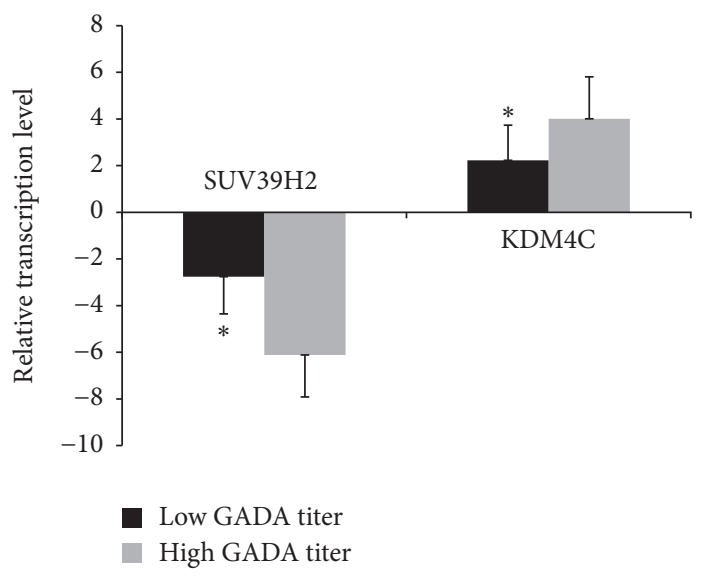

(b)

FIgURE 4: An association of complication and GADA titer with the expression of SUV39H2 and KDM4C in CD4 $4^{+}$T lymphocytes from LADA patients was visible. (a) Relative mRNA levels of histone methyltransferase SUV39H2 and demethylase KDM4C in CD $4^{+} \mathrm{T}$ lymphocytes from LADA patients, ${ }^{*} P<0.05$, LADA patients with complications (LADAc, $n=14$ ) versus LADA patients without complications (LADAnc, $n=12$ ). (b) Relative mRNA levels of histone methyltransferase SUV39H2 and demethylase KDM4C in CD4 ${ }^{+}$T lymphocytes from LADA patients, ${ }^{*} P<0.05$, LADA patients with low GADA titer $(n=17)$ versus LADA patients with high GADA titer $(n=9)$.

was upregulated in LADA patients compared to healthy controls (Figure 3(b)). Other histone methyltransferases and demethylases detected in our study showed no statistical difference between LADA patients and controls. Compared to LADA patients without complication, the gene expression of SUV39H2 was downregulated and KDM4C was upregulated in LADA patients with complication $(P<0.05$, Figure $4(\mathrm{a})$ ). In the meantime, compared to LADA patients with low GADA titer, the gene expression of SUV39H2 was downregulated and KDM4C was upregulated in LADA patients with high GADA titer $(P<0.05$, Figure $4(b))$.

\section{Discussion}

The prevalence of diabetes is increasing year by year. LADA is a subtype of diabetes of which exact pathogenesis is unknown. Histone methylation is a dynamic epigenetic process that participates in a diverse array of cellular processes and has been found to associate with autoimmune diseases, including type 1 diabetes [9-12]. The objective of this study was to explore whether histone methylation was associated with the pathogenesis of LADA. We chose $\mathrm{CD} 4^{+}$ $\mathrm{T}$ lymphocytes to study, and the reason was as follows. Firstly, these cells can be separated from peripheral blood which could be obtained during a regular follow-up. Next and more importantly, although there were many immune cells involved in the pathogenesis of LADA, including $\mathrm{CD}^{+}$ $\mathrm{T}$ lymphocytes, $\mathrm{CD}^{+} \mathrm{T}$ lymphocytes, $\mathrm{B}$ lymphocytes, and monocytes, $\mathrm{CD} 4^{+} \mathrm{T}$ lymphocytes play a central role in the pathogenesis of LADA [3-6].

We demonstrated the global histone 3 lysine 4 and lysine 9 methylation pattern in $\mathrm{CD}^{+}{ }^{+} \mathrm{T}$ lymphocytes from LADA patients. We found that reduced global H3 lysine 9 methylation existed in $\mathrm{CD}^{+}{ }^{+} \mathrm{T}$ lymphocytes from LADA patients, and the reduced $\mathrm{H} 3$ lysine 9 methylation was associated with GADA titer but not significantly correlated with blood glucose (HbAlc). Glutamic acid decarboxylase (GAD) has been recognized as a target antigen in type 1 diabetes. Treatment with GAD in the nonobese diabetes mouse, a model of type 1 diabetes, can prevent diabetes. GAD formulated with aluminum (alum), using in a dose-finding study in LADA, would preserve insulin secretion. GAD autoantibodies (GADA) is undoubtedly one of conditions for a confirmatory LADA diagnosis. In fact, GADA is far from being solely a biomarker but has its specific role in the pathogenesis of LADA. As $\mathrm{H} 3$ lysine 9 methylation was correlated with GADA, we speculated that $\mathrm{H} 3$ lysine 9 methylation may contribute to the pathogenesis of LADA.

Histone methylation, which was regulated by specific histone methyltransferases and histone demethylases, affects chromatin compaction and transcription. Histone methyltransferases and demethylases were promising targets for new drugs for autoimmune diseases [16-19]. Histone 3 lysine 4 methylation was regulated by histone methyltransferase SET1 and histone demethylase LSD1, KDM5A, KDM5B, KDM5C, and KDM5D. Histone 3 lysine 9 methylation was regulated by histone methyltransferase G9a, SUV39H1, SUV39H2, and histone demethylase LSD1, KDM3A, KDM3B, KDM4A, KDM4B, and KDM4C [20, 21]. In this study, we found that the expression of histone methyltransferase $\mathrm{SUV} 39 \mathrm{H} 2$ in LADA patients was downregulated and the expression of histone demethylase KDM4C was upregulated. SUV39H2 and KDM4C were responsible for histone 3 lysine 9 methylation and demethylation. We speculated that the low expression of the SUV39H2 and high expression of KDM4C may explain the reduction of global histone $\mathrm{H} 3$ lysine 9 methylation in the $\mathrm{CD}^{+} \mathrm{T}$ lymphocytes from LADA patients.

We further studied the association of $\mathrm{H} 3$ lysine 9 methylation with complications of diabetes. The results showed that LADA patients with complications had a relatively 
reduced global $\mathrm{H} 3$ lysine 9 methylation compared to LADA patients without complication. HbAlc was related to the progression of complications [22, 23]. We found H3 lysine 9 methylation was correlated with complications of diabetes but not correlated with $\mathrm{HbAlc}$. We speculated that $\mathrm{H} 3$ lysine 9 methylation was an independent factor for the progression of complications of LADA.

Islet transplantation was shown to have positive consequences on diabetic complications [24]. In kidney-pancreas transplanted patients, most of metabolic and functional features of the central nervous system appeared to be near normalized after a 5-year follow-up period of sustained normoglycemia [25]. Possibly, histone H3 lysine 9 methylation lever would be normalized after islet transplantation in LADA patients. It would be valuable to perform such research.

In conclusion, we found a reduced histone $\mathrm{H} 3$ lysine 9 methylation pattern in LADA with low expression of the methyltransferase SUV39H2 and high expression of the demethylase KDM4C.

\section{Conflicts of Interest}

Xi-yu Liu and Hong Li have no conflicts of interest.

\section{Acknowledgments}

This work was supported by the grants from Project of Medical Health and Science and Technology in Zhejiang Province (2016140828; 201473015).

\section{References}

[1] Y. Xu, L. Wang, J. He et al., "Prevalence and control of diabetes in Chinese adults," The Journal of the American Medical Association, vol. 310, no. 9, pp. 948-959, 2013.

[2] Z. Zhou, Y. Xiang, L. Ji et al., "Frequency, immunogenetics, and clinical characteristics of latent autoimmune diabetes in China (LADA China Study): a nationwide, multicenter, clinic-based cross-sectional study," Diabetes, vol. 62, no. 2, pp. 543-550, 2013.

[3] Z. Yang, Z. Zhou, G. Huang et al., "The CD4+ regulatory Tcells is decreased in adults with latent autoimmune diabetes," Diabetes Research and Clinical Practice, vol. 76, no. 1, pp. 126131, 2007.

[4] M. Radenkovic, C. Silver, J. Arvastsson et al., "Altered regulatory $\mathrm{T}$ cell phenotype in latent autoimmune diabetes of the adults (LADA)," Clinical \& Experimental Immunology, vol. 186, no. 1, pp. 46-56, 2016.

[5] N. Marek-Trzonkowska, M. Myśliwiec, A. Dobyszuk et al., "Therapy of type 1 diabetes with CD4+CD25highCD127regulatory $\mathrm{T}$ cells prolongs survival of pancreatic islets-results of one year follow-up," Clinical Immunology, vol. 153, no. 1, pp. 23-30, 2014.

[6] R. Dahan, J. A. Gebe, A. Preisinger et al., "Antigen-specific immunomodulation for type 1 diabetes by novel recombinant antibodies directed against diabetes-associates auto-reactive $\mathrm{T}$ cell epitope," Journal of Autoimmunity, vol. 47, pp. 83-93, 2013.
[7] E. Laugesen, J. A. Østergaard, and R. D. G. Leslie, "Latent autoimmune diabetes of the adult: current knowledge and uncertainty," Diabetic Medicine, vol. 32, no. 7, pp. 843-852, 2015.

[8] B. Gupta and R. D. Hawkins, "Epigenomics of autoimmune diseases," Immunology and Cell Biology, vol. 93, no. 3, pp. 271276, 2015.

[9] L. Haery, R. C. Thompson, and T. D. Gilmore, "Histone acetyltransferases and histone deacetylases in B- and T-cell development, physiology and malignancy," Genes and Cancer, vol. 6, no. 5-6, pp. 184-213, 2015.

[10] X. Li, C. Li, X. Li et al., "Involvement of histone lysine methylation in 21 gene expression in rat kidney in vivo and rat mesangial cells in vitro under diabetic conditions," Journal of Diabetes Research, vol. 2016, Article ID 3853242, 12 pages, 2016.

[11] M. A. Reddy, E. Zhang, and R. Natarajan, "Epigenetic mechanisms in diabetic complications and metabolic memory," Diabetologia, vol. 58, no. 3, pp. 443-455, 2015.

[12] F. Miao, D. D. Smith, L. Zhang, A. Min, W. Feng, and R. Natarajan, "Lymphocytes from patients with type 1 diabetes display a distinct profile of chromatin histone H3 lysine 9 dimethylation: an epigenetic study in diabetes," Diabetes, vol. 57, no. 12, pp. 3189-3198, 2008

[13] F. Miao, Z. Chen, L. Zhang et al., "Profiles of epigenetic histone post-translational modifications at type 1 diabetes susceptible genes," Journal of Biological Chemistry, vol. 287, no. 20, pp. 16335-16345, 2012.

[14] M. Hernandez, A. Mollo, J. R. Marsal et al., "Insulin secretion in patients with latent autoimmune diabetes (LADA): half way between type 1 and type 2 diabetes: Action LADA 9," BMC Endocrine Disorders, vol. 15, no. 1, article 1, 2015.

[15] S. Brophy, H. Davies, S. Mannan, H. Brunt, and R. Williams, "Interventions for latent autoimmune diabetes (LADA) in adults," Cochrane Database of Systematic Reviews, vol. 9, Article ID CD006165, 2011.

[16] J.-Y. Lee, S.-H. Lee, S.-H. Heo et al., "Novel function of lysine methyltransferase G9a in the regulation of Sox2 protein stability," PLoS ONE, vol. 10, no. 10, Article ID e0141118, 2015.

[17] E. Zhao, J. Ding, Y. Xia et al., "KDM4C and ATF4 cooperate in transcriptional control of amino acid metabolism," Cell Reports, vol. 14, no. 3, pp. 506-519, 2016.

[18] L. Morera, M. Lübbert, and M. Jung, "Targeting histone methyltransferases and demethylases in clinical trials for cancer therapy," Clinical Epigenetics, vol. 8, no. 1, pp. 1227-1241, 2016.

[19] C. Hui and T. Ye, "Synthesis of lysine methyltransferase inhibitors," Frontiers in Chemistry, vol. 3, article 44, 2015.

[20] S. Krishnan, S. Horowitz, and R. C. Trievel, "Structure and function of histone $\mathrm{H} 3$ lysine 9 methyltransferases and demethylases," ChemBioChem, vol. 12, no. 2, pp. 254-263, 2011.

[21] E. M. Michalak and J. E. Visvader, "Dysregulation of histone methyltransferases in breast cancer-opportunities for new targeted therapies?" Molecular Oncology, vol. 10, no. 10, pp. 1497-1515, 2016.

[22] S. Genuth, "Insights from the diabetes control and complications trial/epidemiology of diabetes interventions and complications study on the use of intensive glycemic treatment to reduce the risk of complications of type 1 diabetes," Endocrine Practice, vol. 12, supplement 1, pp. 34-41, 2006. 
[23] J. M. Lachin, N. H. White, D. P. Hainsworth, W. Sun, P. A. Cleary, and D. M. Nathan, "Effect of intensive Diabetes therapy on the progression of diabetic retinopathy in patients with type 1 diabetes: 18 years of follow-up in the DCCT/EDIC," Diabetes, vol. 64, no. 2, pp. 631-642, 2015.

[24] R. Bassi and P. Fiorina, "Impact of islet transplantation on diabetes complications and quality of life," Current Diabetes Reports, vol. 11, no. 5, pp. 355-363, 2011.

[25] P. Fiorina, P. Vezzulli, R. Bassi et al., "Near normalization of metabolic and functional features of the central nervous system in type 1 diabetic patients with end-stage renal disease after kidney-pancreas transplantation," Diabetes Care, vol. 35, no. 2, pp. 367-374, 2012. 


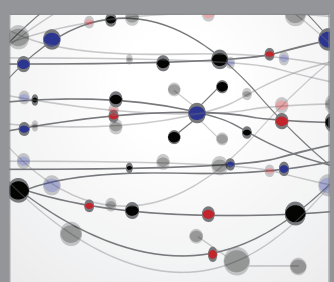

The Scientific World Journal
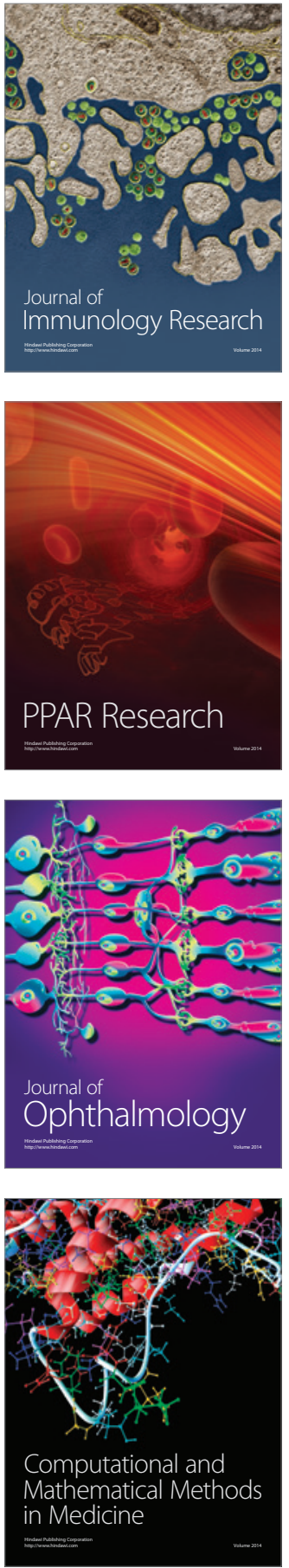

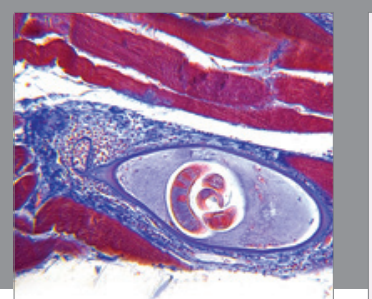

Gastroenterology Research and Practice
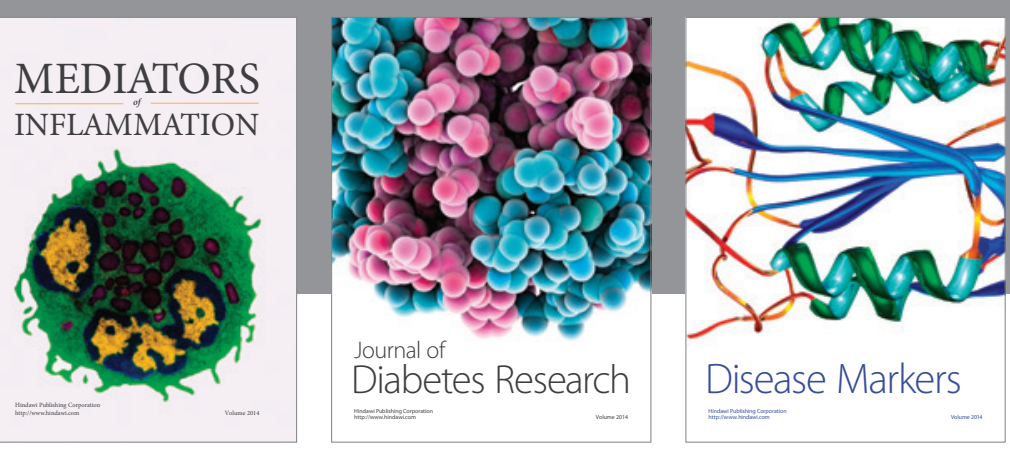

Disease Markers

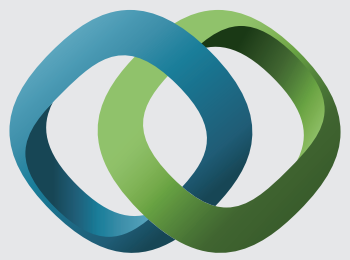

\section{Hindawi}

Submit your manuscripts at

https://www.hindawi.com
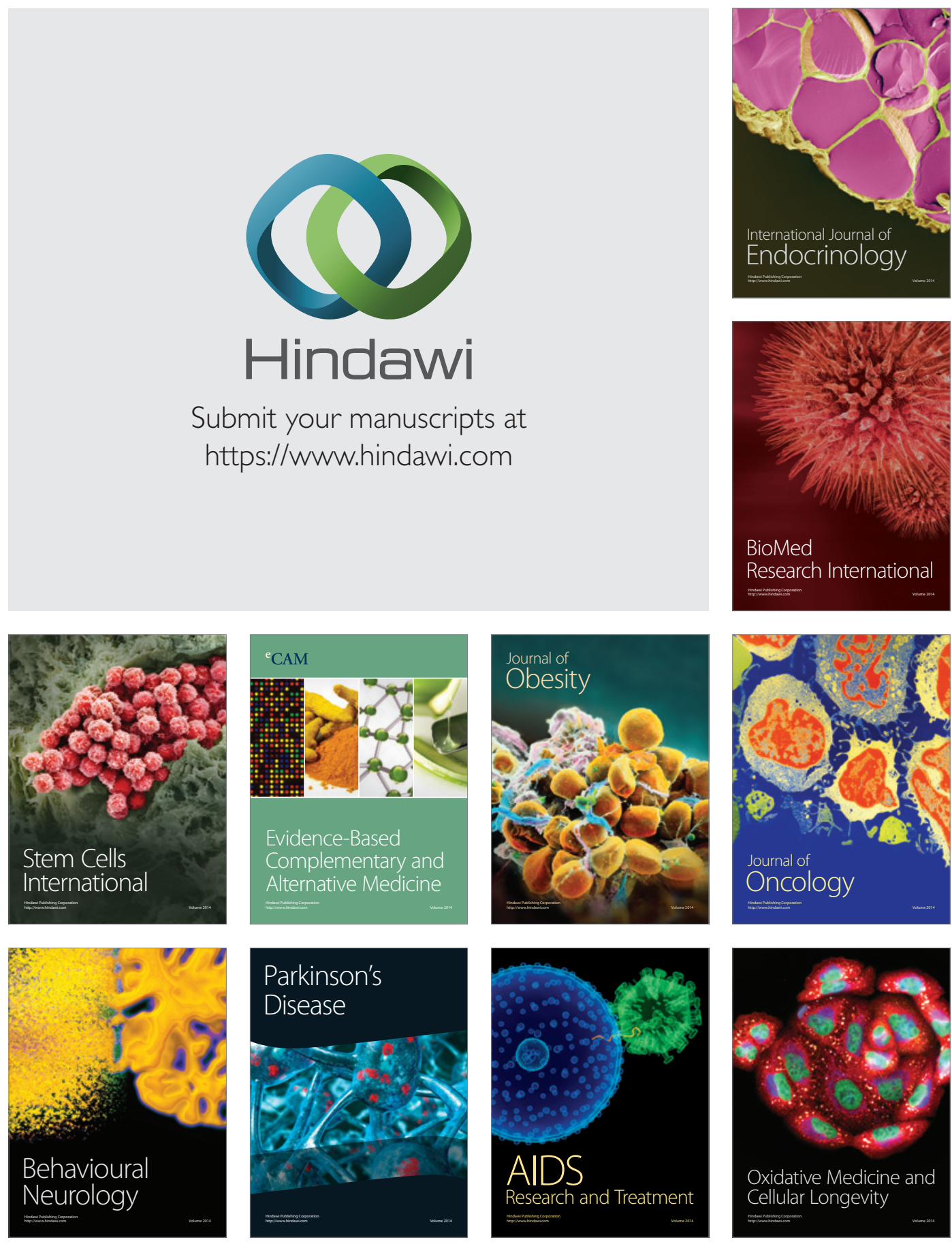